\title{
FUNCIONALIDADE NAS ATIVIDADES INSTRUMENTAIS DE VIDA DIÁRIA EM IDOSOS COM DOENÇA DE ALZHEIMER
}

Functionality in instrumental activities of daily living in elderly with Alzheimer's disease

Funcionalidad en las actividades instrumentales de la vida diaria de los ancianos con enfermedad de Alzheimer

\section{Suzane Cristina Santos ${ }^{* 1}$, Camilla Fagundes de Oliveira Bueno², Juliana Aparecida Wosh ${ }^{3}$, Juliana Sartori Bonini ${ }^{4}$}

${ }^{1}$ Fisioterapeuta, Mestre em Desenvolvimento Comunitário, Universidade Estadual do Centro-Oeste, Guarapuava, Paraná, Brasil.

${ }^{2}$ Fisioterapeuta, Pós-graduada em Fisioterapia Traumato-ortopédica, Universidade Estadual do Centro-Oeste, Guarapuava, Paraná, Brasil.

${ }^{3}$ Fisioterapeuta, Mestre em Desenvolvimento Comunitário, Universidade Estadual do Centro-Oeste, Guarapuava, Paraná, Brasil.

${ }^{4}$ Professora Adjunta e chefe do Laboratório de Neurociência e Comportamento, Universidade Estadual do CentroOeste, Guarapuava, Paraná, Brasil. Presidente da Associação de Estudos, Pesquisas e Auxílio aos Portadores de Alzheimer (AEPAPA), Guarapuava, Paraná, Brasil.

*Correspondência: Laboratório de Neurociência e Comportamento, Universidade Estadual do Centro-Oeste, Alameda Élio Antonio Dalla Vecchia, 838, Guarapuava, PR, Brasil. CEP 85040-167 e-mail suzanecristinaa@gmail.com.

\section{Artigo recebido em 30/04/2020 aprovado em 23/06/2021 publicado em 22/10/2021}

\section{RESUMO}

$\mathrm{O}$ processo de envelhecimento humano traz modificações na capacidade funcional dos idosos. O risco para a incapacidade funcional se agrava quando o idoso é acometido pela Doença de Alzheimer (DA). Nosso objetivo foi avaliar a funcionalidade de idosos com DA por meio das atividades instrumentais de vida diária (AIVDs) e relacionála com o estágio da doença. A amostra foi composta por 56 idosos com DA, para avaliação das AIVDs utilizou-se a Escala de Lawton e Brody e para avaliar o estágio da doença a Escala de Avaliação Clínica da Demência (CDR). Os dados foram apresentados na forma de estatística descritiva. A maior parte dos participantes encontrava-se no estágio grave da doença (CDR3: 41,1\%) e a minoria deles no estágio inicial (CDR1: 23,2\%). Em relação às AIVDs a maioria dos idosos foi classificada com dependência total $(35,7 \%)$, e os idosos no estágio grave da doença foram os que mais apresentaram dependência total $(30,3 \%)$. Apenas idosos do grupo que se encontrava no estágio leve obtiveram classificação de independentes (5,3\%), e nesse mesmo grupo não houve nenhum classificado como dependente total. Em conclusão observa-se que idosos com Doença de Alzheimer apresentam um declínio funcional progressivo na realização de atividades de vida diária.

Palavras-chave: Doença de Alzheimer; Funcionalidade; Atividades de vida diária.

\section{ABSTRACT}

The human aging process brings changes in the functional capacity of the elderly. The risk for functional disability worsens when the elderly are affected by Alzheimer's disease (AD). The aim of this study was to evaluate the functionality of elderly patients with $A D$, with the instrumental activities of daily living (IADL) and relate the functional capacity to the stage of the disease. This study included 56 patients with AD. Their IADLs were assessed using the Lawton-Brody scale, and the disease stage using the Clinical Dementia Rating (CDR). Data were presented as descriptive statistics. Most of the participants were in the severe stage of the CDR (41.1\%) and the minority of 
them were in the mild stage (23.2\%). Regarding IADL most elderly was classified as total dependence (35.7\%), and the elderly in the severe stage of the CDR were the ones that showed total dependence (30.3\%). Only the mild stage group obtained independent rating (5.3\%), and in that group there was no classified as full dependent. In conclusion was observed that elderly people with Alzheimer's disease have a progressive functional decline in performing activities of daily living.

Keywords: Alzheimer's disease; Functionality; Activities of daily living.

\section{RESUMEN}

El proceso de envejecimiento humano trae cambios en la capacidad funcional de los ancianos. El riesgo de discapacidad funcional empeora cuando los ancianos se ven afectados por la enfermedad de Alzheimer (EA). Nuestro objetivo era evaluar la funcionalidad de los ancianos con EA, a través de actividades instrumentales de la vida diaria (IADL) y relacionar la funcionalidad con la etapa de la enfermedad. La muestra consistió en 56 ancianos con EA, se usó la Escala de Lawton y Brody para evaluar las IADL y la Escala de Clasificación de Demencia Clínica (CDR) para evaluar el estadio. Los datos se presentaron en forma de estadística descriptiva. La mayoría de los participantes estaban en la etapa severa de la enfermedad $(C D R 3=41,1 \%)$ y la minoría de ellos en la etapa inicial $(C D R I=23,2 \%)$. En relación con los IADL, la mayoría de los ancianos se clasificó como con dependencia total (35,7\%), y los ancianos en la etapa grave de la enfermedad tuvieron la mayor dependencia total (30,3\%). Solo los ancianos en el grupo que estaba en la etapa leve obtuvieron una clasificación de independientes (5,3\%), y en este mismo grupo no había nadie clasificado como totalmente dependiente. En conclusión, observamos que los ancianos con EA tienen una disminución funcional progresiva en la realización de actividades de la vida diaria.

Descriptores: enfermedad de Alzheimer; Funcionalidad; Actividades de la vida diaria.

\section{INTRODUÇÃO}

O processo de envelhecimento humano é uma condição progressiva que acarreta modificações fisiológicas, bioquímicas e psicológicas, responsáveis por mudanças na capacidade funcional dos idosos. A evolução tecnológica, associada ao aumento da qualidade de vida, tem levado a um envelhecimento cada vez mais ativo e longínquo da população mundial. Nesse quadro, as doenças crônicas e neurodegenerativas recebem destaque, por apresentarem prevalência crescente em nosso meio (FAGUNDES et al., 2017; ARAÚJO et al., 2016).

Dentre elas, as demências possuem destaque como fator importante de morbidade e mortalidade com relevante impacto na diminuição da funcionalidade dos idosos, decorrente do declínio da cognição, o qual observa-se a ocorrência na Doença de Alzheimer (DA), que hoje é um problema de saúde que cresce rapidamente em importância e número (PINHEIRO et al., 2020; LIU-SEIFERT et al., 2018; ARAÚJO et al., 2016).
A DA é uma patologia de etiologia multifatorial caracterizada em sua fisiopatologia por uma neurodegeneração consequente da formação de placas senis, com o acúmulo da proteína beta amiloide $(\beta A)$ e de emaranhados neurofibrilares, que estão relacionados às alterações do estado de fosforilação da proteína tau no espaço cerebral (HASKEL et al., 2017; MONGE-ARGILÉS et al., 2010). As características clínicas dessa demência apresentam-se com perda progressiva de memória e outras habilidades cognitivas, como a atenção, fluência verbal, lucidez e grau de vigília que se deterioram com a evolução da doença (GOES, 2014). Esses sintomas levam ao declínio da capacidade funcional com perda gradual da autonomia, que propicia dificuldades na realização de suas atividades de vida diária, prejudicando o paciente em seu desempenho social e ocupacional (ARAÚJO, et al., 2016; SANTOS E BORGES, 2015).

O risco para a incapacidade funcional se agrava quando o idoso é acometido pela DA, pois a falta de memória e perda das habilidades cognitivas, 
podem interferir negativamente no desempenho funcional. Com o avanço da doença déficits cognitivos associados aos déficits funcionais, como a perda da função motora, com as contraturas musculares, rigidez articular, diminuição do equilíbrio, lentidão dos movimentos e perda da coordenação, contribuem para a diminuição da autonomia e independência, e em consequência, da realização de Atividades Instrumentais de Vida Diária (AIVDs), já que estas são relativamente complexas e requerem bom estado cognitivo e funcional (PINHEIRO et al., 2020; EPPERLY, et al., 2017).

A diminuição da funcionalidade na DA prejudica a qualidade de vida do idoso, pois a perda de autonomia submete-os ao cuidado e supervisão integral de outros, ocasionando também um aumento da sobrecarga desses cuidadores. Dessa forma, avaliar o processo de declínio funcional dessa população é importante para que sejam realizadas intervenções no sentido de minimizar esses efeitos.

Diante do exposto, o presente estudo teve a finalidade de avaliar a funcionalidade nas atividades instrumentais de vida diária de idosos com DA e relacionar a capacidade funcional com o estágio da doença.

\section{MATERIAIS E MÉTODOS}

$\mathrm{O}$ presente estudo caracteriza-se como observacional transversal descritivo (BORDALO, 2006), faz parte de uma investigação mais ampla sobre a Doença de Alzheimer e, foi realizado entre os anos de 2014 e 2015.

A amostra foi composta por idosos com diagnóstico clínico de Doença de Alzheimer identificado por médicos neurologistas ou geriatras, participantes do Programa de Distribuição de Medicamentos Excepcionais do Ministério da Saúde, que recebem medicações específicas para doença fornecida pela $5^{\text {a }}$ Regional de Saúde no município de
Guarapuava, Paraná, Brasil. Foram incluídos no estudo todos os idosos com diagnóstico clínico de Doença de Alzheimer participantes do programa e excluídos os que não tiveram consentimento para participar da pesquisa e aqueles que apresentaram algum item dos testes de avaliação incompleto.

A coleta de dados foi realizada na residência de cada idoso, com a aplicação dos questionários para o idoso e para o seu responsável. A avaliação foi composta por dois questionários, a Escala de Lawton e Brody para avaliação da funcionalidade por meio das AIVDs e a escala para a Avaliação Clínica da Demência (CDR - Clinical Dementia Rating), versão em português, para avaliar o estadiamento da doença. Ambas as avaliações foram aplicadas por profissionais da saúde capacitados para a aplicação.

A Escala de Lawton e Brody avalia oito atividades instrumentais de vida diária: utilização do telefone, realização de compras, preparação das refeições, tarefas domésticas, lavagem da roupa, utilização de meios de transporte, manejo da medicação e responsabilidade de assuntos financeiros (ZIDAN et al., 2012; FIGUEIREDO et al., 2013).

Para cada AIVD o idoso é classificado como dependente ( 0 pontos) ou independente ( 1 ponto). Para os homens não se contabilizam a preparação das refeições, as tarefas domésticas e a lavagem da roupa. A pontuação final é obtida pela soma da pontuação das 8 AIVDs e varia entre 0 a 8 pontos para mulheres e 0 a 5 pontos para o homem, sendo que quanto maior a pontuação maior a independência. O idoso será classificado em dependência total, dependência grave, dependência moderada, dependência ligeira e independente (LAWTON e BRODY, 1969).

A Avaliação Clínica da Demência (CDR) foi desenvolvida para graduar demências, especialmente na DA (HUGHES, 1982). É dividida nas categorias de memória, orientação, julgamento e solução de problemas, assuntos na comunidade, lar e hobbies e 
cuidados pessoais, e para cada uma delas o idoso é classificado em CDR0,5 (demência questionável) CDR1 (demência leve), CDR2 (demência moderada) e CDR3 (demência grave), de acordo com os critérios pré-estabelecidos (GOES et al., 2014).

Os dados coletados das avaliações foram tabulados e armazenados em um banco de dados do Microsoft Excel 2010 e para a análise desses dados foi utilizada a estatística descritiva, com o cálculo da média, desvio padrão e frequências descritas em tabelas.

Este estudo foi aprovado pelo Comitê de Ética em Pesquisa da Universidade Estadual do CentroOeste, sob o Parecer no 611.316/2014. O Termo de Consentimento Livre e Esclarecido (TCLE) foi assinado pelo responsável legal do idoso que participou da pesquisa.

\section{RESULTADOS E DISCUSSÃO}

Foram triados para a pesquisa 64 pacientes, sendo que desses dois dos responsáveis não aceitaram participar do estudo, quatro haviam falecido e dois não foram encontrados no endereço do registro. Assim, a amostra final foi composta por 56 participantes.

A amostra estudada foi composta por indivíduos de ambos os sexos com idade média de $78,6 \pm 8,7$ anos, sendo em sua maioria mulheres. A escolaridade dos idosos apresentou média de $3,92 \pm 2,56$ anos. Em relação ao estágio da doença, a maior parte dos participantes encontrava-se no estágio grave da doença e a minoria no estágio inicial (Tabela 1).

Tabela 1. Características dos participantes

\begin{tabular}{ccc}
\hline CARACTERÍSTICAS & MÉDIA n & DP \% \\
\hline FEMININO (n/\%) & 36 & 64,2 \\
MASCULINO (n/\%) & 20 & 35,7 \\
IDADE & & \\
MULHERES (M \pm ) & 77,4 & 12,56 \\
HOMENS (M \pm ) & 78,6 & 8,82 \\
ESCOLARIDADE (anos) & 3,92 & 2,56 \\
CDR1 (n/\%) & 13 & 23,2 \\
CDR2 (n/\%) & 20 & 35,7 \\
CDR3 (n/\%) & 23 & 41,1 \\
\hline
\end{tabular}

DP: desvio padrão; CDR: Clinical Dementia Rating.

Nos três estágios da doença a prevalência foi de mulheres, no grupo CDR 1 haviam 8 mulheres e 5 homens, CDR 2 as mulheres eram em um total de 13 e homens 7, e no grupo de CDR 3 eram 15 mulheres e 8 homens.

$\mathrm{Na}$ avaliação das AIVDs pela Escala de Lawton e Brody, a pontuação variou entre 0 e 8 com média de 2,23 $\pm 1,96$. Dos idosos que compuseram a amostra $35,7 \% \quad(n=20)$ foram classificados em dependência total, 30,3\% ( $n=17)$ foram classificados em dependência grave, $19,7 \%$ ( $\mathrm{n}=11)$ em dependência moderada, 9\% $(n=5)$ em dependência ligeira e 5,3\% $(n=3)$ classificados como independentes. A maioria dos idosos possuía dependência total e a minoria apresentou independência. 
Dos idosos com dependência total 17 encontravam-se no estágio grave da doença e 3 idosos no estágio moderado; dos idosos classificados em dependência grave, 2 encontravam-se no estágio leve, 9 com demência moderada, e 6 com demência grave; na categoria dependência moderada, 6 dos idosos encontravam-se no estágio de demência moderada e 5 em demência leve; dos cinco idosos com dependência ligeira 3 encontravam-se em demência leve e $2 \mathrm{em}$ moderada; dos 3 idosos classificados como independentes todos estavam classificados como demência leve (Tabela 2).

Tabela 2 - Comparação entre estágio da demência e a funcionalidade

\begin{tabular}{cccc}
\hline AIVDs & CDR1 \%(n) & CDR2 \%(n) & CDR3 \%(n) \\
\hline Dependência total & 0 & $5,3(3)$ & $30,3(17)$ \\
Dependência grave & $3,5(2)$ & $16(9)$ & $10,7(6)$ \\
Dependência moderada & $8,9(5)$ & $10,7(6)$ & $0(0)$ \\
Dependência ligeira & $5,3(3)$ & $3,5(2)$ & $0(0)$ \\
Independente & $5,3(3)$ & $0(0)$ & $0(0)$ \\
\hline
\end{tabular}

CDR: Clinical Dementia Rating; AIVDs: Atividades Instrumentais de Vida Diária.

O objetivo do presente estudo foi avaliar a capacidade funcional de idosos com DA, e relacionála com o estágio da doença. Os resultados mostraram que a maior parte dos idosos com DA possuía dependência total $(35,7 \%)$, bem como os idosos no estágio grave da doença foram os que mais apresentaram dependência total (30,3\%). Dos idosos que se encontravam no estágio leve da demência, não houve nenhum classificado como dependente total.

Estudos sobre funcionalidade na DA têm mostrado que as alterações na capacidade funcional podem ser observadas desde os estágios iniciais da doença, e com o progresso da mesma o declínio no desempenho de atividades diárias é maior (PINHEIRO et al., 2020; LIU-SEIFERT, et al., 2018; PEDROSO et al, 2018; HASKEL et al., 2017; LENARDT et al., 2011).

Uma característica relevante observada na amostra foi o baixo nível de escolaridade, que teve como média 3,92 $\pm 2,56$ anos. O baixo nível educacional é apontado como um provável fator ambiental predisponente à doença, a baixa escolaridade permite ao indivíduo uma menor compensação para o déficit cognitivo (SOUZA et al., 2008; FAGUNDES, et al., 2017).

A maioria dos idosos do estudo apresentou dependência total e apenas uma pequena parte apresentou independência, corroborando com os resultados de um estudo semelhante, que avaliou a funcionalidade de 55 idosos portadores de DA, em que os pesquisadores encontraram apenas um idoso classificado como independente para as AIVDs, 15 idosos apresentaram dependência total e 39 foram classificados como semi dependentes (LENARDT et al., 2011).

No estudo de Lenardt e colaboradores (2011) que avaliou o desempenho de idosos com Alzheimer nas atividades básicas e instrumentais de vida diária, a independência nas AIVDs foi significativamente pior com o agravamento dos sintomas da doença, o que também pode ser observado no presente estudo uma vez que de todos os idosos aqui avaliados houve um maior número de idosos com dependência total no estágio mais avançado da doença (CDR3), bem como nenhum idoso desse grupo foi classificado em dependência moderada, ligeira ou independente, o que 
mostra que com o avanço da doença o idoso torna-se mais dependente.

Um estudo semelhante que avaliou a funcionalidade nas atividades instrumentais e básicas de vida diária em idosos com DA nos três estágios da doença, os autores encontraram uma dependência funcional maior em idosos estadeados em CDR 1 e 3 (HASKEL et al., 2017), assim como os resultados obtidos no presente estudo.

Fagundes e colaboradores (2017) observaram em seu estudo um declínio progressivo na funcionalidade de idosos com DA, sendo que foi identificado maior incapacidade nas AIVDs e as atividades básicas de vida diária preservadas por mais tempo, bem como a perda da funcionalidade também foi associada ao à diminuição da capacidade cognitiva.

Foi identificado nesses resultados que os idosos do grupo CDR1 foram os que obtiveram resultados mais satisfatórios, uma vez que esse foi o único grupo onde identificou-se idosos independentes, e nenhum idoso com dependência total. Um estudo que avaliou a funcionalidade das atividades instrumentais e básicas de vida diária em 74 idosos com DA, a independência foi significativamente pior com o agravamento dos sintomas da doença. Mostrando diferença significativa entre os grupos CDR1 e CDR2 em relação ao CDR3, sendo que o melhor desempenho foi obtido no grupo CDR1 (ZIDAN et al., 2012).

No presente estudo identificou-se três idosos independentes nas atividades instrumentais de vida diária. Haskel e colaboradores (2017) identificaram sete idosos independentes na amostra, sendo que desses 6 encontravam-se em CDR 1 e apenas 1 em CDR 2, identificaram também que o maior número de dependentes total estava no CDR 3. Observa-se que o comprometimento na capacidade funcional de realização das AIVDs é diferente nas fazes da DA tornando-se mais acentuado à medida que o comprometimento cognitivo avança. (HASKEL, et al., 2017; FAGUNDES, et al., 2017; PINHEIRO, et al., 2018).

Independente das condições de risco para o desenvolvimento da DA, o tempo de avanço da demência varia de pessoa para pessoa, e que além do comprometimento cognitivo, a capacidade funcional também pode se apresentar como um dos marcadores da evolução da doença (PEDROSO et al., 2018; LIUSEIFERT, et al., 2018).

Em um estudo em que foram avaliadas as funções executivas, atividades da vida diária e habilidade motora de idosos com doenças neurodegenerativas, os pesquisadores observaram que principalmente nas atividades instrumentais de vida diária os idosos com DA apresentaram pior comprometimento quando comparados a idosos com Doença de Parkinson com demência e sem demência e idosos saudáveis (FELIPPE et al., 2014).

O processo neurodegenerativo da DA leva a uma piora no desempenho das atividades instrumentais da vida diária e aumenta a carga sobre os cuidadores (SANTOS e BORGES, 2015). Além de que, as dificuldades que os pacientes apresentam ao realizar as atividades cotidianas são graves, pois podem causar isolamento e exclusão social deles, interferindo na sua qualidade de vida (PINHEIRO et al., 2020; PEDROSO et al., 2018).

A dependência nas atividades de vida diária devido à demência está associada com comprometimento cognitivo e indica um agravamento do processo de demência (PINHEIRO et al., 2020; FAGUNDES et al., 2017). Também é considerado um fator de risco para o aumento da mortalidade. Idosos com demência e dependência grave e que requer mais assistência durante a execução de atividades da vida diária têm um risco de morte três vezes maior do que de adultos mais velhos que exercem suas atividades normalmente. De acordo com a literatura, os pacientes 
que adiam o início de comprometimento funcional tendem a ter um menor grau de sofrimento devido à demência, minimizar a sobrecarga sobre os cuidadores, e diminuir o risco de mortalidade (PINHEIRO et al., 2020; LIU-SEIFERT et al., 2018; FAGUNDES et al., 2017; NASCIMENTO et al., 2012).

O presente estudo apresenta algumas limitações, tais como: o número reduzido da amostra, avaliação da subjetiva das atividades funcionais funcionalidade, a qual foi realizada por meio de relatos dos cuidadores e não por observação direta de sua realização. Além disso, outros testes de avaliação de atividades instrumentais e básicas de vida diária poderiam ser acrescentados para enriquecer as avaliações.

\section{CONCLUSÃO}

Idosos com Doença de Alzheimer apresentam um declínio funcional progressivo na realização de atividades de vida diária, uma vez que idosos que se encontram no estágio mais avançado da doença apresentam um nível de dependência maior do que aqueles em estágios iniciais.

Esta pesquisa contribui para um melhor entendimento sobre a funcionalidade na Doença de Alzheimer e dos aspectos funcionais na evolução da doença, podendo auxiliar na atuação dos profissionais da saúde junto a essa população.

Sugere-se a realização de novos estudos que explorem de forma mais ampla o tema aqui discutido.

Todos os autores declararam não haver qualquer potencial conflito de interesses referente a este artigo.

\section{REFERÊNCIAS}

ARAUJO, E.S.; GERZSON, L.R.; OLIVEIRA, L.O. Qualidade de vida e sobrecarga: perfil dos cuidadores de idosos com doença de Alzheimer. Cinergis, Santa Cruz do Sul, v.17, n.1, p.27-31, 2016.
BORDALO, A.A. Estudo transversal e/ou longitudinal. Rev. Para. Med., Belém, v.20, n 4, p.5, 2006.

EPPERLY, T.; DUNAY, M.A.; BOICE, J.L.; Alzheimer Disease: Pharmacologic and Nonpharmacologic Therapies for Cognitive and Functional Symptoms. American Family Physician, Leawood, v.95, n.12, p.771-778, 2017

FAGUNDES, T.A.; PEREIRA, A.G.P.; BUENO, K.M.P.; ASSIS M.G. Incapacidade funcional de idosos com demência. Cad. Ter. Ocup. UFSCar, São Carlos, v.25, n.1, p.159-169, 2017

FELIPPE, L.A.; OLIVEIRA, R.T.; GARCIA, M.; SILVA-HAMU, T.C.D.; SANTOS, S.M.S.; CHRISTOFOLETTI, G. Funções executivas, atividades da vida diária e habilidade motora de idosos com doenças neurodegenerativas. J. bras. psiquiatr., Rio de Janeiro, v.63, n.1, p.39-47, 2014.

FIGUEIREDO, C.S.; ASSIS, M.G.; SILVA, S.L.A.; DIAS, R.C.; MANCINI, M.C.; Functional and cognitive changes in community-dwelling elderly: Longitudinal study. Braz. J. Phys. Ther., São Carlos, v.17, n.3, p.297-306, 2013.

GOES, V.F.; MELLO-CARPES, P.B.; OLIVEIRA, L.O.; HACK, J.; MAGRO, M.; BONINI, J.S. Avaliação do risco de disfagia, estado nutricional e ingestão calórica em idosos com Alzheimer. Rev. Latino-Am. Enfermagem, Ribeirão Preto, v.22, n.2, p.317-324, 2014.

GURE, T.R.; LANGA, K.M.; FISHER, G.G.; PIETTE, J.D.; PLASSMAN, B.L. Functional limitations in older adults who have cognitive impairment without dementia. J Geriatr Pshychiatry Neurol., Michigan, v.26, n.2, p.78-85, 2013.

HASKEL, M.V.L.; BONINI, J.S.; SANTOS, S.C.; SILVA, W.C.F.N.; BUENO, C.F.O.; BORTOLANZA, M.C.Z; DANIEL, C.R. Functionality on mild, moderate and severe Alzheimer's dis-ease: a cros-sectional study. Acta Fisiátrica, São Paulo, v.24, n.2, p. 82-85, 2017.

HUGHES, C.P.; BERG, L.; DANZIGER, W.L.; COBEN, L.A.; MARTIN, R.L. New clinical scale for the staging of dementia. British Journal of Psychiatry, London, v.140, p.566-572, 1982.

LAWTON, M.P.; BRODY, E.M. Assessment of older people: self-maintaining and instru-mental activities of daily living. Gerontologist, New York, v.9, n.3, p.179-186, 1969. 
LENARDT, M.H.; SILVA, S.C.; SEIMA, M.D.; WILLING, M.H.; FUCHS, P.A.O. Desempenho das atividades de vida diária em idosos com Alzheimer. Cogitare Enferm., Curitiba, v.16, n.1, p.13-21, 2011.

LIU-SEIFERT, H.; SIEMERS, E.; SUNDELL, K.; MYNDERSE, M.; CUMMINGS, J.; MOHS, R.; AISEN, P. Analysis of the Relationship of Cognitive Impairment and Functional Impairment in Mild Alzheimer's Disease in EXPEDITION 3. The Journal of Prevention of Alzheimer's Disease JPAD, France, v.5, n.3, p.184-187, 2018.

MEDEIROS, M.E.; GUERRA, R.O. Tradução, adaptação e análises das propriedades psicométricas do activities of daily living questionnaire (ADLQ) para avaliação funcional de pacientes com a doença de Alzheimer. Rev Bras Fisioter., São Carlos, v.13, n.3, p.257-266, 2009.

MONGE-ARGILÉS， J.A.; MUÑOZ-RUIZ， C.; PAMPLIEGA-PÉREZ, A.; GÓMEZ-LÓPEZ, M.J.; SÁNCHEZ-PAYÁ, J.; RODRÍGUEZ BORJA, E.; RUIZ-VEGARA, M.; MONTOYA-GUTIÉRREZ, F.J.; LEIVA-SANTANA, C. Biomarkers in the cerebrospinal fluid of patients with mild cognitive impairment: a meta-analysis of their predictive capacity for the diagnosis of Alzheimer's disease. Rev Neurol., Barcelona, v.50, n.4, p.193-200, 2010.

NASCIMENTO, C.M.C.; TEIXEIRA， C.V.L.; GOBBI, L.T.B.; GOBBI, S.; STELLA, F. A controlled clinical trial on the effects of exercise on neuropsychiatric disorders and instrumental activities in women with Alzheimer's disease. Rev Bras Fisioter, São Carlos, v.16, n.3, p.197-204, 2012.
PEDROSO, R.V.; CORAZZA, D.I.; ANDREATTO, C.A.A.; SILVA, T.M.V.; COSTA, J.L.R.; SANTOSGALDURÓZ, R. F. (2018). Cognitive, functional and physical activity impairment in elderly with Alzheimer's disease. Dement. neuropsychol, v.12, n.1, p.28-34.

PINHEIRO, H.A.; BRANDÃO, J.D.M.; SILVA, A.L. Correlation between functionality, mobility and risk of falls in elderly people with Alzheimer's disease. Rev. bras. cineantropom. desempenho hum., Florianópolis, v.22, e70219, 2020.

RAJAN, K.B.; HEBERT, L.E.; SCHERR, P.A.; MENDES DE LEON, C.F.; EVANS, D.A. Disability in basic and instrumental activities of daily living in associated with faster rate of decline in cognitive function o folder adults. J Gerontol A Biol Sci Med Sci., v.68, n.5, p.624-30, 2013.

SANTOS M.D.; BORGES S.M. Percepção da funcionalidade nas fases leve e moderada da doença de Alzheimer: visão do paciente e seu cuidador. Rev. Bras. Geriatr. Gerontol., Rio de Janeiro, v.18, n.2, p.339-349, 2015.

SOUZA P.A.; SÁ, S.P.C.; BASTOS, R.C.S.; CASSIANO, K.M.; SANTANA, R.F. Oficinas de estimulação cognitiva para idosos com demência: uma estratégia de cuidado na enfermagem gerontológica. Rev Gaúcha Enferm., Porto Alegre, v.29, n.4, p.588595, 2008.

ZIDAN, M.; ARCOVERDE, C.; ARAÚJO, N.B.; VASQUES, P.; RIOS A.; LAKS, J.; DESLANDES, A. Alterações motoras e funcionais em diferentes estágios da doença de Alzheimer. Rev Psiq Clín., São Paulo, v.39, n.5, p.161-165, 2012. 